\title{
EDUCACIÓN INTERCULTURAL, ATENCIÓN A LA DIVERSIDAD Y CALIDAD DE LA FORMACIÓN INICIAL DOCENTE*
}

\author{
INTERCULTURAL EDUCATION, ATTENTION TO DIVERSITY \\ AND QUALITY OF INITIAL TEACHER TRAINING
}

\author{
Carlos Mondaca Rojas**, Eugenio Sánchez Espinoza***
}

\begin{abstract}
En los últimos años, problemáticas significativas se han manifestado en el sistema educativo nacional e internacional impactando de forma importante en sus distintos niveles. Temáticas como la gratuidad, el acceso universal, la diversidad cultural, la migración, la inclusión, el enfoque de género, el feminismo, las diferencias socioeconómicas y los entornos desfavorecidos de donde provienen generalmente los estudiantes, son coyunturas que ya se encuentran al interior de las instituciones educativas y que deben ser tratadas con la profundidad que requieren para estar acorde a los tiempos de complejidad que estas presentan. Si sumamos las problemáticas internas de las instituciones educativas, podemos señalar que atentan contra los indicadores de calidad; sobre todo aquellos que son medidos en pruebas estandarizadas internacionales y nacionales que dan cuenta de resultados desiguales en las trayectorias educativas de personas pertenecientes a los grupos más vulnerables, como los mencionados anteriormente (OCDE, 2016b).

Entre los factores que explican los bajos resultados en los indicadores de calidad a nivel nacional que tienen relación con la diversidad en todos sus componentes, se visibilizan desafíos a futuro en materia de política educacional, en el trabajo y la enseñanza de lenguas originarias y extranjeras, la necesidad de armonizar y contextualizar el currículum monocultural y asimilacionista, incorporar el enfoque de género, la pertinencia étnica, estrategias de inclusión efectiva, así como el enfoque intercultural y el desarrollo de metodologías pedagógicas que ayuden a disminuir la brecha de los sectores vulnerables en temas como la deserción, el abandono escolar, la titulación efectiva, entre otras. Todos
\end{abstract}

estos componentes dan cuenta del impacto negativo e insuficiente de las políticas educativas, y además indican falencias en la calidad de la educación para atender las problemáticas que emergen a partir de los diferentes tipos de diversidad que existen en la actualidad en nuestras instituciones educativas (Geeregat, Vásquez y Fierro, 2012).

Estos déficit de indicadores de calidad en educación a nivel de países en vías de desarrollo como el nuestro, tiene orígenes diversos: las nuevas reformas educativas, el cambio en la carrera docente, el mercado y las condiciones laborales de remuneraciones bajas y agobio laboral, la evaluación y encasillamiento docente, entre otros factores, hacen cada vez menos atractiva la profesión docente (OCDE, 2016c). Todos estos factores son determinantes en los estándares de calidad en la formación inicial de profesores en materia de atención de las diversidades. Un indicador de esto se observa en los resultados de la Prueba INICIA aplicada a más de 5.000 egresados de enseñanza básica y secundaria entre el 2008 y 2014. Dichos resultados muestran que los futuros profesores tenían un dominio muy por debajo de lo que deberían saber acerca de contenidos disciplinarios y pedagógicos. Esto resulta problemático, pues uno de los desafíos que impone la OCDE (2014) para la educación es que los establecimientos estén mejor preparados en todos sus componentes para atender las diversidades. Esto supone como condición necesaria una formación adecuada de los futuros profesores en las instituciones de Educación Superior (IES). Sin embargo, la evidencia internacional muestra que trabajar con poblaciones estudiantiles diversas es una de las áreas donde los docentes se sienten menos preparados (OCDE, 2014), lo que

\footnotetext{
* El presente artículo es resultado del Proyecto FONDECYT N 1160976 . Se agradece el apoyo del Convenio Marco Formación Inicial Docente, UTA1756.

** Universidad de Tarapacá. Departamento de Educación. Arica, Chile. Correo electrónico: cemondacar@uta.cl

*** Universidad de Tarapacá. Departamento de Ciencias Históricas y Geográficas. Arica, Chile. Correo electrónico: esanchez@ uta.cl
} 
ha sido corroborado también a nivel del norte de Chile (Mondaca, et al., 2018). En este sentido, es relevante evaluar y analizar el rol de la diversidad cultural en los procesos de implementación de la formación inicial de profesores (Gaete, Gómez y Bascopé, 2016)

Además, resulta tremendamente necesario analizar de manera sistemática las buenas prácticas de las IES de Chile en FID (OCDE, 2016a), con el fin de contribuir a la preparación de profesores con competencias interculturales adecuadas a sus propios contextos locales de diversidad. Ciertamente, una FID de calidad permitirá establecer bases sólidas para generar una política pública que sea capaz de responder adecuadamente a los desafíos y tensiones que los contextos de diversidad imponen al sistema escolar. Esto redundaría en una sociedad más justa, solidaria e inclusiva.

El desarrollo de una FID que incorpore adecuadamente el componente de las diversidades, finalmente permitiría que en su formación los profesores adopten una postura cívica y de ciudadanía activa contra todas las formas de discriminación y exclusión; y que satisfagan las necesidades de los alumnos de diversos orígenes, impartiendo valores fundamentales comunes transversales que prevengan el racismo y la intolerancia, como lo exponen algunos de los trabajos publicados en este número.

El panorama actual de nuestro país demanda el diseño e implementación de políticas públicas orientadas a mejorar la calidad de los docentes. De allí que se hayan realizado un número importante de investigaciones acerca de la FID, las que han hecho propuestas significativas para aumentar los estándares de calidad (García, 2018). Es importante destacar que se observa en este ámbito el interés tanto público como privado para el mejoramiento de la calidad de la enseñanza en ámbitos educativos formales.

Lo anterior se refleja en que en el último tiempo esta orientación ha sido una prioridad del Ministerio de Educación, la que se traduce en una mejora de la oferta declarada y lo que efectivamente se entrega en FID, pues como indican los magros resultados en evaluaciones -que se mencionan más arriba- existe en este punto todavía una tensión no resuelta. Respecto de esto, la OCDE $(2004 ; 2005)$, el Consejo Asesor Presidencial (2006) y el Informe Final de Evaluación Programa Fomento a la Calidad de la Formación Inicial Docente (2015) advertían del bajo nivel de preparación de los futuros profesores.
Este diagnóstico ha sido abordado a partir de la instalación entre 1998 y 2002 del Programa de Fortalecimiento de la Formación Inicial Docente; y entre el 2004 y 2007 del Programa de Mejoramiento de la Calidad y Equidad de la Educación Superior. En la misma línea, en el último tiempo se ha implementado un financiamiento concursable denominado Convenios de Desempeño para la Formación Inicial de Profesores y durante los últimos dos años en el financiamiento basal mediante Convenios Marco de Formación Inicial Docente.

De esto se desprende que estas políticas para fortalecer la FID no han sido mínimas y que existe consenso respecto de la importancia de la formación de los profesores. Sin embargo, desde el ámbito académico y de investigación científica falta un mayor desarrollo y estudio del área tanto a nivel nacional como internacional (Consejo Asesor Presidencial, 2006) del impacto de las políticas de FID en las IES; y es todavía más significativo el déficit de producción científica respecto de FID y diversidad cultural. Al respecto, los estudios de Shulman (1986) y Darling-Hammond (1998; 2000) confrontan la investigación científica respecto del dominio disciplinar y pedagógico, y las competencias que un futuro profesor debiera tener. Con todo, estos autores concluyen que pese al dominio de estos ámbitos esto no significa que su posterior desempeño docente se deba únicamente a su formación en las universidades y quiénes y cómo los formaron o deformaron como docentes, ya que la diversidad cultural es importante para la FID (Zapata, 2014).

Sin duda, y como se indica en la literatura, la formación inicial docente es la primera y crucial etapa del desarrollo profesional de todo futuro profesor, que mediante un currículum articulado entre cursos de educación, pedagogía, disciplinas, didácticas, metodologías y prácticas, entre otros elementos, se preparan y forman en programas que generalmente duran de cuatro a cinco años, adquiriendo las competencias necesarias para enseñar a sus futuros estudiantes (Jiménez y Montecinos, 2018; Tardiff, 2010; Day, 2005; Tey y Gustems, 2016). Para Zabala (citado en Morong, 2017), a la FID se le demanda que la enseñanza debe estar centrada en el futuro profesor en su proceso de enseñanza-aprendizaje, para que este evidencie calidad educativa. Es por ello que la FID ha tenido varias evaluaciones orientadas a medir tal exigencia y calidad de los docentes, entre ellas, la prueba Inicia, el Marco 
de la Buena Enseñanza y los diseños curriculares tendientes a fomentar habilidades al amparo de un modelo educativo constructivista.

Un antecedente que toda FID debe considerar es lo señalado en el Informe McKinsey (McKinsey citado en Barber y Mourshed, 2008), realizado por encargo de la OCDE. Este documento tiene por objetivo comprender por qué los sistemas educativos con más alto desempeño del mundo alcanzan resultados mejores que la mayoría de los demás, y por qué ciertas reformas educativas tienen tanto éxito, cuando muchas otras no logran su cometido. Se concluye que el éxito depende de:

1) conseguir a las personas más aptas para ejercer la docencia,

2) desarrollarlas hasta convertirlas en instructores eficientes, $y$

3) garantizar que el sistema escolar sea capaz de brindar la mejor instrucción posible para los educandos.

En términos histórico-contextuales, la formación inicial docente en Chile tiene como hito fundacional la creación de la primera Escuela Normal en 1842, y a partir de 1889 la Universidad de Chile comienza a impartir las carreras pedagógicas para enseñanza secundaria creando el Instituto Pedagógico (Ávalos, 2002). Desde entonces, cada gobierno fue incorporando sus propias perspectivas y buscó el aumento en la cobertura. Sin embargo, desde entonces el sistema educativo chileno ha operado desde el presupuesto de la existencia de una sociedad homogénea y de una comunidad imaginada que nace desde los modelos históricos y cívicos con que se construyó el Estado-Nación sustentado en una ideologización de una sola cultura e identidad nacional bajo el estereotipo de superioridad esencializado por la Guerra del Pacífico.

En esta particular zona de la frontera norte, aún en pleno siglo XXI, existe una marcada resistencia en el ámbito político y educacional en la implementación de un enfoque intercultural e inclusivo en el sistema educativo. Por lo general, se presta poca atención a las necesidades de aprendizajes de los futuros profesores desde el currículum y desde las prácticas docentes en IES, excluyendo la alteridad, al homogeneizar a todos en una mirada nacional chilena. Un caso paradigmático de esto es el programa de educación intercultural bilingüe, que más que avanzar hacia la inclusión de la diversidad cultural y a transformar una estructura nacionalista, tiende a reforzar la asimilación (Mondaca, 2018).
Junto con esto, una característica fundamental de la situación chilena es la diversidad de programas de formación de profesores, con distintos niveles de calidad, con diferentes enfoques en donde difícilmente se puede hacer una caracterización general de los mismos, al punto de que se ha llegado a hablar de carencia de un sistema de educación superior propiamente tal (Ávalos, 2014). La actual política hacia la educación superior se inscribe en el modelo de acreditación. Para esto se han creado los organismos estatales pertinentes (Comisión Nacional de Acreditación de Programas de Pregrado-CONAPP). Para finalizar, es admisible señalar que los lineamientos estratégicos de las políticas educativas o de la política docente desde hace al menos 15 años se han insertado, surgido y construido en el debate político-educativo respecto de las maneras de entender a los docentes, los académicos, la formación inicial, los procesos de cambio, las instituciones educativas y el rol del Estado y no en sus componente principales, en este caso la diversidad cultural.

En este contexto, en la presente edición de Diálogo Andino, Revista de Historia, Geografía y Cultura Andina, encontraremos artículos posicionados en Latinoamérica con énfasis en los países andinos que abordan temas reconocedores de una sociedad diversa. De este modo, en el $\mathrm{N}^{\circ} 57$ se divulgan investigaciones relacionadas al campo de una educación intercultural, la formación inicial docente, las relaciones e intercambios de saberes de Chile y sus países vecinos, así como migraciones actuales y exilios y asilos producidos en décadas pasadas, entre otros.

En primer lugar, Juan Beltrán y Sergio Pérez nos describen aquellos factores que dificultan la relación entre la educación escolar y el saber y conocimiento mapuche dentro de las escuelas en la zona costera de La Araucanía, evidenciando la escasa incorporación de conocimientos de la cultura mapuche en el currículo escolar y revelando la ausencia de un enfoque educativo intercultural.

Seguidamente, se exhibe un trabajo que analiza el grado de desarrollo de las competencias interculturales en los estudiantes en práctica de las carreras de pedagogía de la Universidad de Tarapacá, ubicada en la frontera de Norte de Chile. Si bien se miden las características a este nivel de los estudiantes en aulas y establecimientos culturalmente diversos, también se considera el autorreporte de los estudiantes en práctica acerca de sus propias percepciones 
de lo que ha sido su formación inicial. En síntesis, se evidencian ciertas carencias en el ámbito de la educación, que al parecer no solo tiene que ver con falta de competencias pedagógicas que apunten a la interculturalidad, sino también con factores estructurales como el posicionamiento del desarrollo de la práctica al final de la carrera.

En esta misma línea, María Guadalupe Ñeco sugiere la importancia de integrar en distintas instituciones educativas la formación del profesorado en el enfoque educativo intercultural. Coloca el foco de análisis en la formación del profesorado de una institución de carácter público, la Universidad Veracruzana de México, concluyendo que el profesorado estudiado reconoce la falta de programas y planes que resignifiquen lo comprendido respecto de lo intercultural en su formación teórica, y que en cuanto a las prácticas pedagógicas, concluye la autora, todo se basa en un punto de vista academicista. Ñeco también evidencia en sus resultados la existencia de un quehacer docente individualizado, en el sentido de no contacto con los otros, fuera del dinamismo cultural y social que nos circunda.

Posteriormente se ahonda en una educación intercultural, y ampliando el contexto a países latinoamericanos promotores de una enseñanza intercultural, Omar Turra, Mauricio Lagos y Mario Valdés nos presentan un estudio en el que analizan la identidad promovida por la enseñanza histórica como parte de la formación escolar mediante una revisión de contenidos de currículum escolares. Nuevamente, este trabajo esclarece en sus resultados hechos como la tendencia a un predominio de un concepto de identidad homogenizadora, que pese a acuerdos respecto de incluir la diversidad cultural indígena en el sistema educativo, se continúa con una educación monocultural.

Por otra parte, atendiendo a la dimensión lingüística que actualmente despierta interés en distintas investigaciones debido al aumento y diversidad de flujos migratorios, por ejemplo de poblaciones no hispanoparlantes, Diálogo Andino incluye un artículo que muestra las herramientas insuficientes de la población local para responder a las demandas lingüísticas que presenta uno de los grupos migrantes, como es la población haitiana. En efecto, identifica los elementos pedagógicos base de un programa de competencias comunicativas interculturales como la adopción de un enfoque de reciprocidad de lenguas y una adecuada selección de los saberes culturales, entre otros.
Dando una continuidad temática con lo anterior, Iskra Pavez y Caterine Galaz indagan en otra faceta de la vida de la población migrante: la relacionada a los derechos de sus hijos e hijas. $\mathrm{Al}$ respecto, visualizan las posibilidades efectivas de ejercer sus derechos como nueva ciudadanía a nivel social. Se focaliza así este trabajo en la niñez evidenciando brechas de desigualdad destacando el ámbito educativo y social.

Por otra parte, se presenta un trabajo que propone que es preciso desarrollar una imaginación espacial más allá de lo territorial. De esta forma, Romina Ramos expone que existen expresiones no territoriales que practican los cruzadores fronterizos, las que se traducen en los frecuentes viajes de un lado a otro del límite fronterizo ubicado entre la región de Tarapacá, Chile, y la provincia de Oruro, Bolivia.

Ligado al espacio de frontera, el artículo de Dilla y Álvarez explora el surgimiento de complejos urbanos transfronterizos en América Latina específicamente de Tacna y Arica, estudiando cómo estos circuitos generan transferencias de valores de una parte a la otra, dando lugar a un proceso de intercambio desigual favorable a la ciudad chilena.

Un siguiente artículo analiza ciertos eventos que configuran la relación bilateral entre los países de Chile y Bolivia. Incluye las interpretaciones bolivianas que responden a su constante aspiración marítima en su política exterior hacia Chile. Los autores presentan una explicación a base de la teoría que sustenta la idea de "Imaginario Social Marítimo Boliviano", en la que se aprecia cómo las sociedades crean y recrean sus interpretaciones de la realidad.

Luego, el foco se traslada a la postura del Estado chileno apreciable en la narración y discurso histórico que ha sostenido frente a temas de relación y conflicto con los Estados vecinos de Bolivia y Perú. A su vez se aprecia un análisis de la problemática que se genera cuando una narración histórica con ciertos valores tradicionales como el patriotismo y la heroicidad militar se enfrentan a los paradigmas del mundo actual.

Seguidamente, nuestra revista expone un artículo que explora un Chile de la década de los setenta, en un contexto de régimen político y social de dictadura militar, donde se analizan las situaciones de exilio, asilo y refugio conforme con ciertos movimientos de personas, específicamente el caso de los ciudadanos bolivianos que luego del golpe militar de Hugo Bánzer se dirigieron a Chile en busca de protección siendo acogidos por 
el gobierno de Salvador Allende, pero que poco tiempo después debieron partir a un nuevo refugio para evitar caer bajo la represión dirigida durante los primeros años de la dictadura militar chilena.

Finalmente, se proporciona un trabajo que aborda el intercambio epistolar entre el escritor José María Arguedas y el dirigente campesino Hugo Blanco, ambos voceros de los sectores indígenas del Ande de Perú, quienes intercambiaron tres cartas escritas en quechua, que destacan aspectos claves que, según el autor, configuran un momento significativo de la historia política peruana reciente y la construcción de su identidad étnica.

Por último, se acota aún más el contexto explorado en Latinoamérica exhibiendo un trabajo etnográfico realizado con base en una comunidad campesina de Huancavelica, en los Andes centrales de Perú. Se exponen las nociones que tienen los comuneros y comuneras de aquella localidad en torno a lo que entienden por "comer bien" y cómo estas formas de alimentación están ligadas a las labores que realizan a diario en el campo. En un espacio donde patologías de origen alimenticio, como anemia y desnutrición, aquejan a los campesinos. El artículo propone indagar en las semejanzas y diferencias de los modelos interpretativos de la comunidad autóctona y los planes internacionales de nutrición que intervienen en Perú, con el fin de lograr planes complementarios.

\section{Referencias Citadas}

Ávalos, B.

2002 Profesores para Chile: Historia de un Proyecto. Santiago: Ministerio de Educación.

Ávalos, B.

2014 "La formación inicial docente en Chile: Tensiones entre políticas de apoyo y control". Estudios Pedagógicos (Valdivia), 40 (Especial), 11-28. Https://dx.doi.org/10.4067/ S0718-07052014000200002

Barber. M. y Mourshed, M.

2008 Cómo hicieron los sistemas educativos con mejor desempeño del mundo para alcanzar sus objetivos. Disponible en: http://www.mckinsey.com/clientservice/socialsector/ resources/pdf/Worlds_School_Systems_Final.pdf

Consejo Asesor Presidencial para la Calidad de la Educación 2006 Informe Final de Consejo Asesor Presidencial para la Calidad de la Educación. Santiago. Recuperado: 2012. http://mt.educarchile.cl/MT/jjbrunner/archives/libros/ ConsejoAsesor/Inf_def.pdf

Darling-Hammond, L.

1998 "Teachers and teaching: Testing policy hypotheses from a national commission report". Educational Researcher, 27(1), 5-15.

Darling-Hammond, L.

2000 Reforming teacher preparation and licensing: Debating the evidence, Teachers College Record, 102, 5-27.

Day, C.

2005 Formar docentes. Cómo, cuándo y en qué condiciones aprende el profesorado. Narcea S.A. Ediciones Madrid.

Gaete, A.; Gómez, V. y Bascopé, M.

2016 ¿Qué le piden los profesores a la formación inicial docente en Chile? Temas de la Agenda Pública. Año 11 / No 86 / mayo 2016. ISSN 0718-9745.

García P.; Jihan, Cordero A. y Graciela, R.

2018 "Motivaciones para ingresar a la formación docente. Revisión de estudios empíricos publicados en el siglo XXI". Perspectiva Educacional (Valparaíso), Vol. 50, $\mathrm{N}^{\circ}$ 2 doi:10.4151/07189729-Vol.57-Iss.2-Art.727

Geeregat, O.; Vásquez, O. \& Fierro, J.M.

2012 "Procesos de formación inicial docente en contextos multiculturales: inclusión y exclusión”. Estudios Pedagógicos
(Valdivia), 38(1), 345-351. https://dx.doi.org/10.4067/ S0718-07052012000100021

Informe Final de Evaluación Programa Fomento a la Calidad de la Formación Inicial Docente

2015 http://www.dipres.gob.cl/597/articles-141238_informe_final.pdf

Jiménez, F y Montecinos, C.

2018 "Diversidad, modelos de gestión y formación inicial docente: desafíos formativos desde una perspectiva de justicia social". Rev. Bras. Educ. vol.23 Rio de Janeiro 2018 Epub 11Dic-2017. http://dx.doi.org/10.1590/s1413-24782018230005

Mondaca, C.; Muñoz, W.; Gajardo, Y.; Gairín, J.

2018 "Estrategias y prácticas de inclusión de estudiantes migrantes en las escuelas de Arica y Parinacota, frontera norte de Chile". Revista Estudios Atacameños. Estud. atacam. no.57 San Pedro de Atacama. Páginas: 181-201. http://dx.doi.org/10.4067/S0718-10432018005000101

Mondaca, C.

2018 Educación y migración transfronteriza en el norte de Chile: procesos de inclusión y exclusión de estudiantes migrantes peruanos y bolivianos en las escuelas de la región de Arica y Parinacota. Tesis para optar al Grado de Doctor en Educación. Universidad Autónoma de Barcelona.

Morong, G.; Merino. E. y Arellano, A.

2017 "Características, percepciones y motivaciones de los estudiantes que ingresan a Pedagogía en Chile: el caso de la Universidad Bernardo O'Higgins". Revista Brasileira de Educação, 22(71), e227166. Epub 13 de novembro de 2017.https://dx.doi.org/10.1590/s1413-24782017227166 OCDE

2004 Exámenes de las Políticas Nacionales de Educación. Chile. París: Publicación de la OCDE.

OCDE

2005 Los docentes son importantes: Atraer, formar y conservar los docentes eficientes. División de Política de Educación y Formación de la Dirección de Educación. Publicación de la OCDE.

OCDE

2014 The Governance of Regulators, OECD Best Practice Principles for Regulatory Policy, OECD Publishing, París, http://dx.doi.org/10.1787/9789264209015-en. 
OCDE

2016a Skills matter, Chile. Recuperado de: https://www. oecd.org/skills/piaac/Skills-Matter-Chile.pdf

OCDE

2016b Governance of Regulators' Practices: Accountability, Transparency and Co-ordination. The Governance of Regulators, OECD Publishing, París, http://dx.doi. org/10.1787/9789264255388-en.

\section{OCDE}

2016c Driving Performance at Latvia's Public Utilities Commission. The Governance of Regulators, OECD Publishing, París, http://dx.doi.org/10.1787/9789264257962-en.

Shulman, L. S.

1986 "Those who understand: Knowledge growth in teaching". Educational Researcher, 15(2), 4-14.
Tardiff, M.

2010 Los saberes del docente y su desarrollo profesional. Narcea S.A. Ediciones Madrid.

Tey, A. y Gustems, J.

2016 "El profesorado en la formación de maestros y su incidencia en el carácter". En La formación del carácter de los maestros. Francisco Esteban (ed). Pedagogías UB. Universidad de Barcelona, pp. 89-97.

\section{Zapata, P.}

2017 'The power of saying the normally 'unsaid' as an act of empowering a woman's voice in the academia and the fictional parallel side behind this power in a global era". Qualitative Inquiry, 23(5), pp. 352-354. 